\title{
Secondary Structure and Side-Chain Chromophore Orientation of Isotactic Poly(methacrylamide)s in Solid Film
}

\author{
Lin Fan, ${ }^{*}$ Takayuki Fukada, Masahiko AnNaKa, \\ Masako YoshIKUNI, and Takayuki NAKAHIRA ${ }^{\dagger}$ \\ Department of Applied Chemistry, Faculty of Engineering, Chiba University, \\ Yayoi-cho 1-33, Inage-ku, Chiba 263-8522, Japan \\ * Graduate School of Science and Technology, Chiba University, \\ Yayoi-cho 1-33, Inage-ku, Chiba 263-8522, Japan
}

(Received November 13, 1998)

\begin{abstract}
Intramolecular hydrogen bonding among the side-chain amide groups in isotactic poly[( $S)-1-(1-n a p h t h y l)-$ ethyl methacrylamide] (1) and poly $[(S)$-1-phenylethyl methacrylamide] (2) were examined in films cast from 1,2-dichloroethane (EDC). Curve fitting of the amide I band shows that the peaks for the full hydrogen bonds are broader as compared with those in EDC solution, suggesting that the hydrogen bonds formed in film are looser and less extensive than those in solution. X-Ray diffraction (XRD) measurements show that these polymers give hexagonal-like aggregates in film, indicating that they form rod-like helical structures as suggested by molecular mechanics calculations. Isotactic 2 film shows a positive circular dichroic (CD) peak at $c a .230 \mathrm{~nm}$, characteristic of the fully hydrogen-bonded helical structure, which disappears upon annealing. Isotactic 1 film shows two CD positive peaks at $c a .210 \mathrm{~nm}$ and at $c a .230 \mathrm{~nm}$, their relative intensities changing upon annealing, which together with the CD measurements in solution suggests that the hydrogen-bonded and non-hydrogen bonded structures of isotactic 1 give, in the ${ }^{1} \mathbf{B}_{b}$ band of the naphthyl group, exciton coupling of positive chirality and that of negative chirality, respectively, the overall $\mathrm{CD}$ being determined by their relative contents.

KEY WORDS Poly(methacrylamide)s / Side-Chain Chromophore / Orientation / Secondary Structure / Intramolecular Hydrogen Bonding / Circular Dichroism /
\end{abstract}

Polymers having aromatic and heteroaromatic chromophores in the side chains have been examined extensively as excitation energies as well as electrons can be transported through the pendant chromophores along the polymer chains. ${ }^{1-9}$ If excitation energy or electron transport through the chromophores is not interrupted by ground-state and/or excited-state traps such as dimers or excimers, we will have "molecular wires" which will be useful for molecular devices to be used in the future computers. However, in these studies, except a few, ${ }^{4-6}$ the polymer structures were not sufficiently well designed to efficiently suppress trap formation among the chromophores. We now know that the trap formation is not only affected by the chromophore structure ${ }^{7,8}$ but also by the stereoregularity of the main chain, ${ }^{8,9}$ both of which are important in controlling the total polymer conformation and thus the side-chain chromophore orientation.

Previously, we have prepared isotactic and atactic poly(methacrylamide)s, e.g., poly[(S)-1-(1-naphthyl)ethyl methacrylamide] (1) and poly[(S)-1-phenylethyl methacrylamide] (2), where aromatic groups are introduced in the side chains via amide linkage, and examined how the stereoregularity, the side-chain structure, and the solvent affect the hydrogen bonding interactions among the side-chain amide groups. ${ }^{10,11}$ The IR and ${ }^{1} \mathrm{H}$ NMR showed that the isotactic polymers form extensive intramolecular hydrogen bonding among the side-chain amide groups in non-hydrogen bonding solvents such as chloroform and 1,2-dichloroethane (EDC) while the corresponding atactic ones do so only partially. Accordingly, isotactic and atactic 1 gave markedly different $C D$ spectra in EDC, reflecting the difference in side-

$\dagger$ To whom correspondence should be addressed. chain naphthyl group orientation. Isotactic 1 showed rather strong $\mathrm{CD}$ signals, suggesting the formation of a regular secondary structure, i.e., a helical structure of one prevailing handedness with a specific chromophore orientation.

In the present work, we analyzed the IR and CD spectra of isotactic $\mathbf{1}$ and $\mathbf{2}$ in film and examined how the intramolecular hydrogen bonding and the side-chain chromophore orientation are affected, in solid film, by the stereoregularity, the side-chain chromophore structure, and the heat treatment.

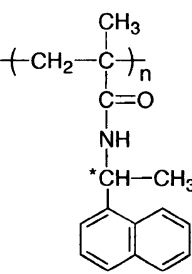

1

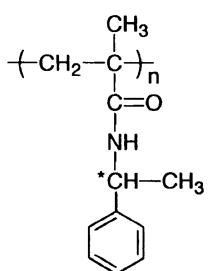

2

\section{EXPERIMENTAL}

\section{Polymers}

Isotactic $\mathbf{1}$ and isotactic $\mathbf{2}$ were prepared by amidation of the polymethacrylic acid obtained by hydrolyzing poly(methyl methacrylate) of an isotactic triad content of larger than $99 \%$ and a degree of polymerization (DP) of 440 as reported previously. ${ }^{10}$ Atactic 1 was prepared similarly using poly(methyl methacrylate) of isotactic, heterotactic, and syndiotactic triad contents of 2,32 , and $64 \%$, repectively, and DP of 700 . The amidation was confirmed to be close to $100 \%$ by elemental analysis and ${ }^{1} \mathrm{H}$ NMR. 
Atactic 2 was prepared by radical polymerization of the corresponding monomer at $30^{\circ} \mathrm{C}$ in tetrahydrofuran (THF) using 2,2'-azobis(4-methoxy-2,4-dimethylvaleronitrile) as an initiator. The isotactic, heterotactic, and syndiotactic triad contents were found to be $10,40,50 \%$, respectively, by ${ }^{1} \mathrm{H}$ NMR.

\section{Measurements and Calculations}

The film samples were prepared by casting the EDC solution on a Teflon plate or on a quartz plate. They were dried in vacuo to constant weights at room temperature. The IR spectra were recorded on a JASCO JIR-7000 FT-IR spectrophotometer by using the $\mathrm{KBr}$ pellet method. Curve fitting was performed with the peak fitting module of Origin software (Microcal Software, Inc., New York). The Voigt function, a sum of Gaussian and Lorentzian band shapes, was used as a fitting function.

DSC measurements were carried out using a MAC Science DSC 3100 S differential scanning calorimeter. The heating scan rate was $10^{\circ} \mathrm{C} \mathrm{min}{ }^{-1}$.

CD and UV spectra were recorded on a JASCO J-500 circular dichrograph and a HITACHI U-3210 spectrophotometer, respectively. The $\mathrm{CD}$ measurements of the films were performed by rotating the sample by $45^{\circ}$ for each measurement. The obtained spectra were then averaged.

Wide-angle X-ray diffraction measurements were performed on the films cast from EDC using a MAC Science MXP X-ray diffractometer with $\mathrm{Cu}-K_{\alpha}$ radiation.

Molecular mechanics calculations were performed on the 24-mers of each polymer using a CAChe molecular mechanics program with an augmented force field for aromaticity. ${ }^{12}$ The conformation is characterized primarily by two main-chain and four side-chain dihedral angles. The calculation was started by freely moving all parts of the initial conformation. The calculations were repeated until the two main-chain and four side-chain dihedral angles became virtually the same for each repeating unit.

Theoretical CD calculations were carried out according to the method reported by Harada ${ }^{13}$ using the geometries of the neighboring chromophores in the lowenergy conformations obtained by molecular mechanics calculations. The exciton wavenumbers were taken from the UV spectra. The transition dipole moment parameters were obtained from the literature. ${ }^{5}$

\section{RESULTS AND DISCUSSION}

Our previous studies have shown that the side-chain amide groups of the poly(methacrylamide)s examined form hydrogen bonds intramolecularly in EDC: their IR spectra do not change in the concentration range of $0.01-0.5 \mathrm{M}$ (in terms of repeating unit). ${ }^{10}$ Curve fitting shows that there are three absorptions in the region of carbonyl stretching vibration (amide I band) with maxima at $c a .1620,1640$, and $1670 \mathrm{~cm}^{-1}$, which are attributed to fully hydrogen-bonded, partially hydrogen-bonded, and free amide carbonyl, respectively (Figure 1). The fully hydrogen-bonded carbonyl absorption of isotactic 2 (Figure 1b) gives, as compared with that of isotactic 1 (Figure 1a), a sharper peak at a slightly lower wave-

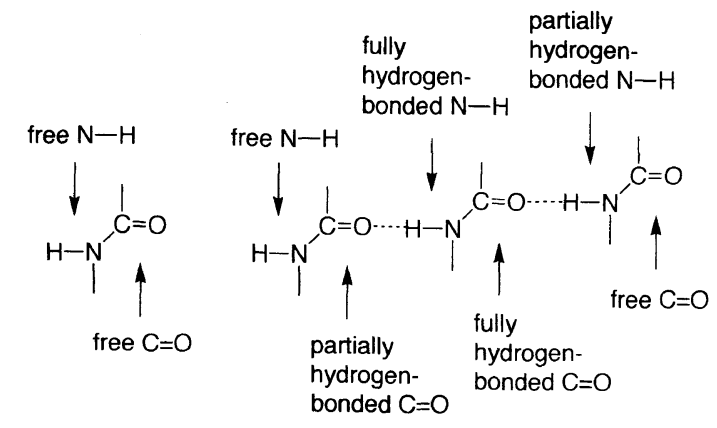

Scheme 1. Hydrogen bonding of side-chain amide groups.

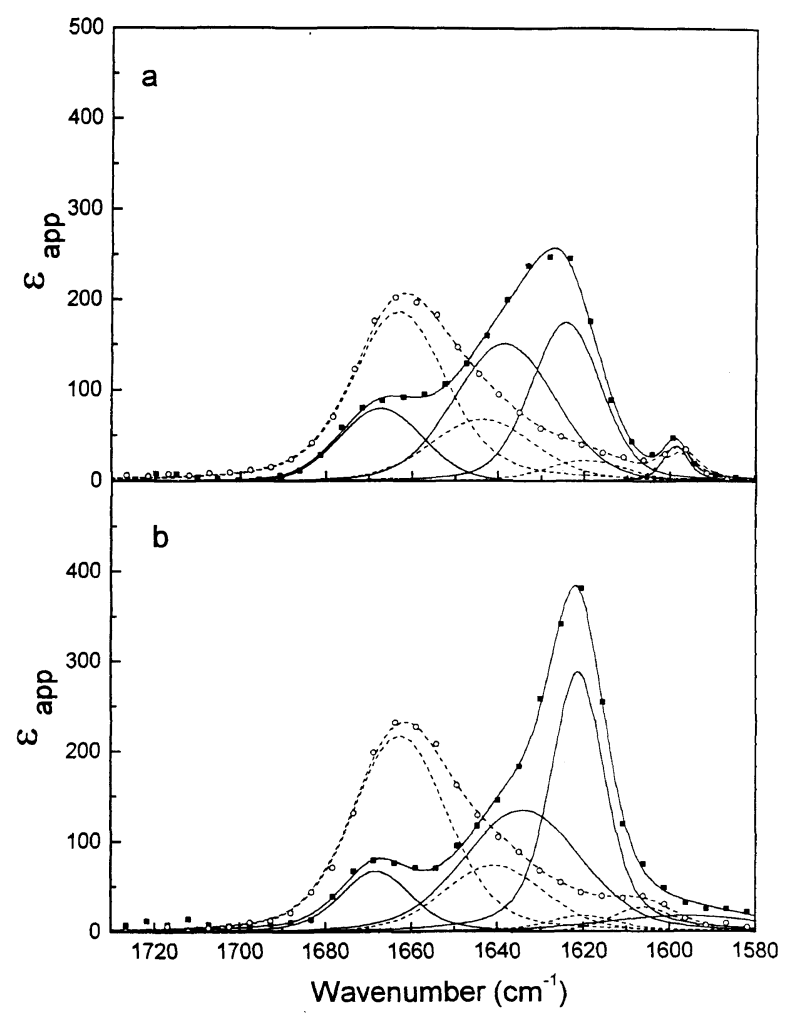

Figure 1. Curve fitting of amide I band of (a) $0.05 \mathrm{M}$ isotactic ( $\square$ ( )

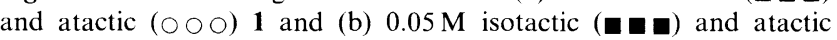
$(O \circ)) 2$ in EDC. The deconvoluted peaks and convoluted spectra of isotactic $\mathbf{1}$ and $\mathbf{2}(-)$ ) and those of atactic $\mathbf{1}$ and $\left.\mathbf{2}(-)_{-}\right)$are also shown.

number, indicating that the full hydrogen bonds formed in isotactic $\mathbf{2}$ are tighter than those in isotactic $\mathbf{1}$. The amide I absorptions of atactic $\mathbf{1}$ and $\mathbf{2}$ are markedly different from those of the corresponding isotactic ones: a peak at $1620 \mathrm{~cm}^{-1}$ is barely noted, and most of the absorption is found at $c a .1665 \mathrm{~cm}^{-1}$ together with some absorption at $c a .1640 \mathrm{~cm}^{-1}$, indicating that most of the carbonyl groups are free from hydrogen bonding.

The amide $I$ bands of isotactic 1 and $\mathbf{2}$ films cast from EDC solutions are broader as compared with those in EDC (Figures 2 and 3); curve fitting shows that each deconvoluted peak is broader and shifted to the higher wavenumber by $5-10 \mathrm{~cm}^{-1}$. The broadness of the peaks for the full and partial hydrogen bonds is apparently due to a wider distribution of geometries of the hydrogen bonds formed between the amide $\mathrm{N}-\mathrm{H}$ and carbonyl ${ }^{14}$; the shift to the higher wavenumber, as in the case of isotactic 2, and the lower relative area of the fully 


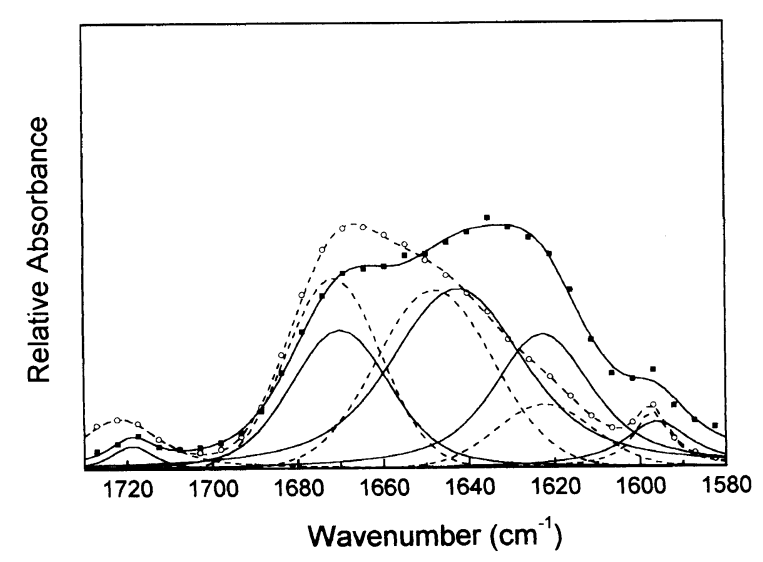

Figure 2. Curve fitting of amide I band of isotactic 1 (घqu) and atactic $1(\circ \bigcirc \bigcirc)$ films cast from EDC. The deconvoluted peaks and convoluted spectra of isotactic 1 (- - ) and those of atactic 1 (-- ) are also shown.

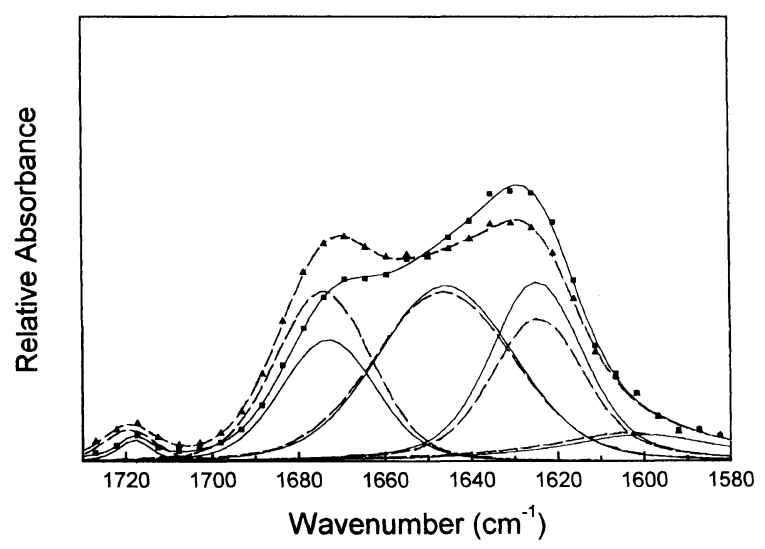

Figure 3. Curve fitting of amide I band of isotactic 2 film before $(\boldsymbol{\square} \boldsymbol{\nabla})$ and after $(\boldsymbol{\Delta} \boldsymbol{\Delta} \boldsymbol{\Delta})$ annealing at $200^{\circ} \mathrm{C}$ for $1 \mathrm{~h}$. The deconvoluted peaks and convoluted spectrum before $(-)$ and after (--) annealing are also shown.

hydrogen-bonded carbonyl peak, suggest that hydrogen bonding in film is looser and less extensive compared with that in solution. Intermolecular interactions in solid film apparently restrict the freedom of movement of the side-chain amide groups to adopt adequate geometries ${ }^{15}$ for formation of the hydrogen bonds or for stabilization of the hydrogen bonds once they are formed. On the other hand, the amide I absorption of atactic 1 film, when compared with that in EDC, shows that the intermolecular interactions lead to slightly more hydrogen bonding among the side-chain amide groups (Figure 2).

The IR spectrum of isotactic 2 underwent a change upon annealing at $200^{\circ} \mathrm{C}$ as shown in Figure 3. The absorption at $\mathrm{ca} .1675 \mathrm{~cm}^{-1}$ increased while the absorption at $c a .1625 \mathrm{~cm}^{-1}$ decreased, indicating that part of the fully hydrogen-bonded structure is destroyed upon annealing.

The glass transition temperature $\left(T_{\mathrm{g}}\right)$ of isotactic $\mathbf{2}$, $159^{\circ} \mathrm{C}$, is much higher than that of an ordinary methacrylate polymer, e.g., $44^{\circ} \mathrm{C}$ of isotactic poly(benzyl methacrylate), due to the reduction of the main-chain mobility by hydrogen bonds among the side-chain amide groups. The $T_{\mathrm{g}}$ decreases by $c a$. $5^{\circ}$ upon annealing at $200^{\circ} \mathrm{C}$, which is consistent with the assumption that

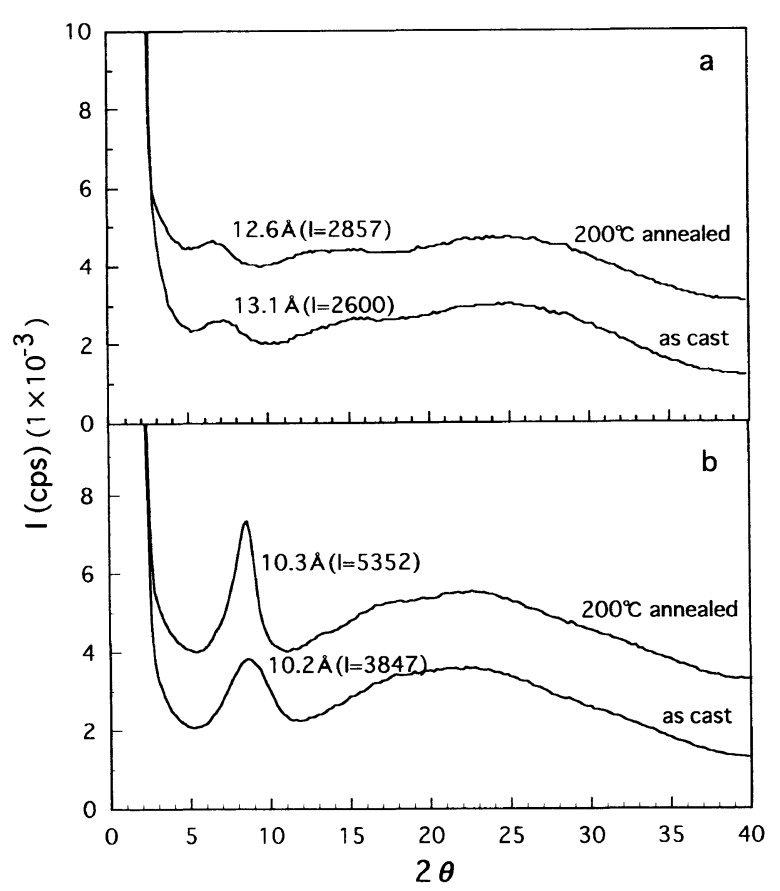

Figure 4. X-Ray diffraction of (a) isotactic 1 and (b) isotactic 2 films before and after annealing at $200^{\circ} \mathrm{C}$ for $1 \mathrm{~h}$.

breaking of the hydrogen bonds in the amorphous region leads to a lower $T_{\mathrm{g}}$.

The $T_{\mathrm{g}}$ of isotactic 1 and that of atactic 1 are 171 and $151^{\circ} \mathrm{C}$, respectively. These $T_{\mathrm{g}}$ 's do not change appreciably. upon annealing at $200^{\circ} \mathrm{C}$. In accord with this, the IR spectra of isotactic and atactic 1 change only slightly upon annealing (not shown). In fact, slight increases of full and partial hydrogen bonding are noted. The larger aromatic chromophores appear to provide a steric effect to prevent the destruction of hydrogen bonds once they are formed.

As shown in Figure 4, the X-ray diffraction of isotactic 1 and 2 gives peaks other than that in the amorphous region, similar to the $\mathrm{X}$-ray diffraction patterns observed with helical poly(L-glutamates). ${ }^{16}$ No such peaks are observed with atactic 1 and $\mathbf{2}$ films. Thus, the isotactic polymers, at least in part, are rod-like and pack themselves side by side to form hexagonal-like aggregates. As isotactic 2 has less bulky side-chain chromophores as compared with isotactic $\mathbf{1}$, the former gives a shorter distance between the polymer chains.

When isotactic $\mathbf{1}$ film is annealed, little change in the $\mathrm{X}$-ray diffraction (XRD) pattern is observed. Isotactic 2 , on the other hand, shows an increase of the peak intensity upon annealing, suggesting an improved packing of polymer chains.

The CD spectrum of isotactic 2 film shows a small positive peak at $c a .230 \mathrm{~nm}$ in addition to a relatively large negative peak in the ${ }^{1} \mathrm{~L}_{\mathrm{a}}$ band of the phenyl group (Figure 5b). The spectrum is similar to that observed in EDC (insert in Figure 5b). The positive peak at $c a$. $230 \mathrm{~nm}$, which we assigned to the $n-\pi *$ absorption band of the side-chain amide groups in the fully hydrogenbonded structure in solution, ${ }^{11}$ however, is weaker than that in EDC. As the IR spectrum suggests, some of the fully hydrogen-bonded structures are destroyed during film formation. When the film is annealed, the peak at 


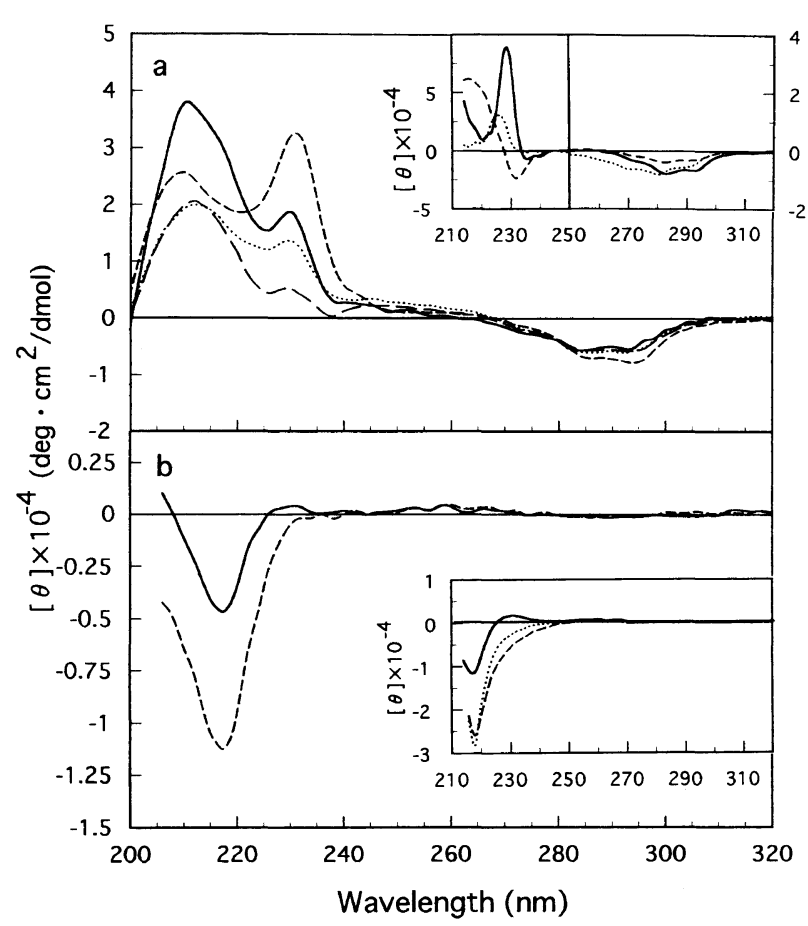

Figure 5. CD spectra of (a) isotactic 1 and (b) isotactic 2 films before $(-)$ and after $(---)$ annealing at $200^{\circ} \mathrm{C}$ for $1 \mathrm{~h}$. The CD spectra of atactic 1 before (- - - ) and after (…..) annealing are also shown in (a). Inserts are the CD spectra of isotactic (-) and atactic (--) 1 (a) and 2 (b) and those of the corresponding monomeric model compounds (….) in EDC.

ca. $230 \mathrm{~nm}$ diminishes and only a negative peak at ca. $218 \mathrm{~nm}$ is observed, giving a CD spectrum resembling that of the monomeric model compound in EDC. The virtual disappearance of the $230-\mathrm{nm}$ peak is in accord with the IR and DSC observations that some of the fully hydrogen bonds are broken upon annealing, but it appears to contradict the XRD observation that annealing improves the packing of the polymer chains. It is suggested either that the $230-\mathrm{nm}$ peak is associated with the fully hydrogen-bonded structure in the amorphous region, which in part breaks down upon annealing, or that the side-chain chromophore orientation in the crystalline region changes significantly upon annealing while the fully hydrogen-bonded structure is maintained. We are yet to know the actual cause for this apparent contradiction.

The CD spectrum of isotactic 1 film (Figure 5a) shows, in addition to a negative peak at $c a .290 \mathrm{~nm}$ in the ${ }^{1} \mathrm{~L}_{\mathrm{a}}$ band of the naphthyl group, two positive peaks at $c a$. $230 \mathrm{~nm}$ and at $c a .210 \mathrm{~nm}$ in the ${ }^{1} \mathrm{~B}_{\mathrm{b}}$ band region. (As shown in the insert, isotactic 1 in EDC gives a rather large positive peak at $c a .230 \mathrm{~nm}$ in the ${ }^{1} \mathrm{~B}_{\mathrm{b}}$ band of the naphthyl group. The CD peak due to the $n-\pi *$ amide transition appears to be buried in this large CD signal. In addition, the $C D$ signal in the ${ }^{1} \mathrm{~L}_{\mathrm{a}}$ band, when compared with that of the monomeric model compound, shows a contribution of exciton coupling of negative exciton chirality. ${ }^{11}$ ) The intensities of the two peaks in the ${ }^{1} B_{b}$ band of isotactic 1 film change upon annealing: the peak at $c a .210 \mathrm{~nm}$ decreased while that at $c a .230 \mathrm{~nm}$ increased. The negative peak at $c a .290 \mathrm{~nm}$ also increases at the cost of the CD in the $270-280 \mathrm{~nm}$ region. Interestingly, while atactic 1 in EDC gives a positive peak

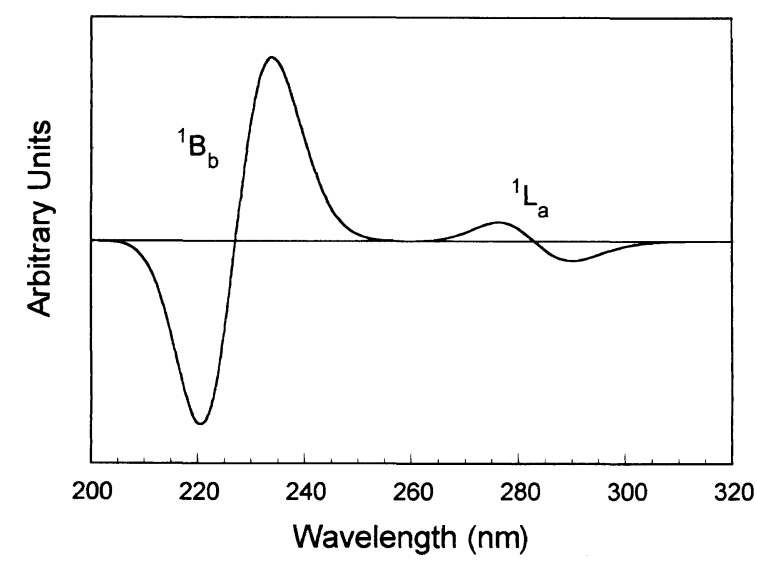

Figure 6. Theoretical $\mathrm{CD}$ for the fully-hydrogen-bonded righthanded helical structure of isotactic 1 .

at $c a .220 \mathrm{~nm}$ and a negative peak at $c a .230 \mathrm{~nm}$, it in film gives a positive peak at $c a .230 \mathrm{~nm}$ in accord with the increase of partial hydrogen bonding observed by IR, and the intensity of that peak increases upon annealing as is the case with isotactic 1. As only slight changes in IR are observed with both isotactic and atactic 1 films upon annealing, the annealing effect is more clearly seen in $C D$, reflecting subtle changes in the orientation of the side-chain chromophores.

We presume that the fully and partially hydrogenbonded structures of isotactic and atactic 1 give, in the ${ }^{1} \mathrm{~B}_{\mathrm{b}}$ band of the naphthyl group, a positive Cotton effect at ca. $230 \mathrm{~nm}$ and, possibly, a negative Cotton effect around $220 \mathrm{~nm}$, i.e., an exciton coupling of positive exciton chirality. The non-hydrogen-bonded structure, which is assumed to be present in part even in isotactic 1 in EDC, apparently gives an exciton coupling of negative exciton chirality in the ${ }^{1} \mathrm{~B}_{\mathrm{b}}$ band of the naphthyl group. The relative contents of these structures would determine the overall $\mathrm{CD}$ spectrum in solution as well as in film.

As reported previously, ${ }^{11}$ molecular mechanics calculations suggest that the fully hydrogen-bonded structures of isotactic 1 and 2 are right-handed $c a .5_{2}$ helices, full hydrogen bonding occurring among every other repeating unit, i.e., $i$ and $i+2$, along the polymer chain. Partial hydrogen bonds are also possible in isotactic $\mathbf{1}$ and $\mathbf{2}$, involving $i$ and $i+2$ repeating units as in the fully hydrogen bonded structure. (No fully hydrogen-bonded helices are allowed for the syndiotactic sequences which are apparently present to a significant extent in atactic polymers; only partial hydrogen bonding, involving $i$ and $i+2$ repeating units, appears to be allowed.) Based on the right-handed fully hydrogen-bonded structure suggested by molecular mechanics calculation, ${ }^{11}$ the theoretical $C D$ for fully hydrogen-bonded isotactic 1 was evaluated by simply considering the two neighboring naphthyl groups in the $i$ and $i+2$ repeating units along the polymer chain. ${ }^{13} \mathrm{~A} \mathrm{CD}$ spectrum with chiralities consistent with those suggested experimentally, i.e., exciton coupling of positive exciton chirality in the ${ }^{1} \mathbf{B}_{b}$ band and that of negative exciton chirality in the ${ }^{1} \mathrm{~L}_{a}$ band, is obtained (Figure 6). Essentially the same exciton coupling is also suggested between $i$ and $i+2$ repeating units in the partially hydrogen-bonded structure. Calcu- 
lations also suggest that rather stable secondary structures free from hydrogen bonding can exist for isotactic as well as syndiotactic sequences, which will allow neighboring naphthyl groups to undergo exciton interaction of negative exciton chirality in the ${ }^{1} \mathbf{B}_{b}$ band. The determination of the exact non-hydrogen-bonded structure awaits further study.

\section{CONCLUSION}

The intramolecular hydrogen bonding among the sidechain amide groups in isotactic $\mathbf{1}$ and $\mathbf{2}$ were investigated in solid film. The IR spectra show that the intramolecular hydrogen bonds in film are weaker, with a broad distribution, than those in solution. XRD measurements show, however, that these polymers give hexagonal-like aggregates in film, indicating that they, at least in part, form rod-like helical structures suggested by molecular mechanics calculations.

Annealing gave different effects on isotactic $\mathbf{1}$ and $\mathbf{2}$. The IR spectra show that the hydrogen-bonded structures in isotactic $\mathbf{2}$ are destroyed in part, while those in isotactic $\mathbf{1}$ are not very much affected.

The CD spectrum of isotactic 2 film shows a CD signal due to the fully hydrogen-bonded structure, part of which is broken down by annealing. Isotactic 1 film shows two $\mathrm{CD}$ peaks attributed to the hydrogen-bonded structure and to the non-hydrogen-bonded structure, the relative contents of these structures changing upon annealing.

Acknowledgments. This work was supported in part by a Grant-in-Aid for Scientific Research (Grant No.
08651045) from the Ministry of Education, Science, Sports and Culture of Japan.

\section{REFERENCES}

1. S. E. Webber, Chem. Rev., 90, 1469 (1990).

2. T. Nakahira, S. Ishizuka, S. Iwabuchi, and K. Kojima, Macromolecules, 16, 297 (1983).

3. A. Itaya, K. Okamoto, and S. Kusabayashi, Bull. Chem. Soc. Jpn., 50, 22 (1977).

4. M. Sato, M. Yoshimoto, T. Nakahira, and S. Iwabuchi, Makromol. Chem., Rapid Commun., 14, 179 (1993).

5. M. Sisido, S, Egusa, and Y. Imanishi, J. Am. Chem. Soc., 105, 4, $1041(1983)$

6. H. H. Fox and M. A. Fox, Macromolecules, 28, 4570 (1995).

7. T. Nakahira, T. Sakuma, S. Iwabuchi, and K. Kojima, J. Polym. Sci., Polym. Phys. Ed., 20, 1863 (1982).

8. T. Ishii, T. Handa, and S. Matsunaga, J. Polym. Sci., Polym. Phys. Ed., 17, 811 (1979).

9. T. Uryu, H. Ohkawa, and R. Oshima, Macromolecules, 20, 712 (1987).

10. T. Nakahira, Fan Lin, C. T. Boon, T. Karato, M. Annaka, M. Yoshikuni, and S. Iwabuchi, Polym. J., 29, 701 (1997).

11. T. Nakahira, L. Fan, C. T. Boon, T. Fukada, T. Karato, M. Annaka, and M. Yoshikuni, Polym. J., 30, 910 (1998).

12. S.-Y. Liu and G. D. Purvis, III, "CAChe Molecular Mechanics Augmented Force Field," Tektronix. Inc., Beaverton, Oregon, 1991.

13. N. Harada and K. Nakanishi, in "Circular Dichroic Spectroscopy-Exciton Coupling in Organic Stereochemistry," Tokyo Kagaku Dozin, Tokyo, 1982, p 217.

14. D. J. Skrovanek, S. E. Howe, P. C. Painter, and M. M. Coleman, Macromolecules, 18, 1676 (1985).

15. G. C. Pimentel and A. L. McClellan, "The Hydrogen Bond," W. H. Freeman and Co., San Francisco, CA., 1969, p 296.

16. J. Watanabe, K. Imai, R. Gehani, and I. Uematsu, J. Polym. Sci., Polym. Phys. Ed., 19, 653 (1981). 